Research Article

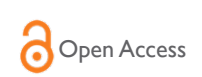

A) CrossMark

\title{
Report on food allergies \& intolerances
}

\begin{abstract}
This report discusses the various issues related to food allergies and intolerances and the current recommendations and guidelines.

Food and adequate nutrition is an essential part of our survival and health however with anecdotal and research evidence it appears that the prevalence of food intolerances and allergies may be on the rise. Although intolerances and allergies can be extremely detrimental to our health and in some cases even fatal, assessing the incidence rates prove to be quite difficult. According to Hadley this may be as a result of differing societal and scientific definitions and problems with diagnosis. ${ }^{1}$ Despite this the FSAI estimate that "between $2-4 \%$ of our population have a clinically diagnosed allergic reaction to certain foods, with the highest prevalence in children". ${ }^{2}$ What may not be included in these figures are the less threatening but also serious food intolerances which can also have serious implications on health
\end{abstract}

Keywords: allergy, food
Volume 2 Issue 4 - 2015

\section{Laurann O Reilly}

Nutritionist, Public Health Nutrition, Ireland

Correspondence: Laurann O Reilly, Nutritionist, MD of Personal Nutrition \& Health, Public Health Nutrition, Ireland, Email lauranndeniseoreilly@gmail.com

Received: April 13,2015 | Published: September 10, 2015
Abbreviations: WAO, world allergy organisation; WUN, world universities network; INDI, irish nutrition and dietetic institute; IBS, irritable bowel syndrome; GMO, genetically modified; EFSA, european food safety authority; NGCS, non-celiac gluten sensitivity; SPT, skin prick tests

\section{Food allergies \& intolerances}

From an immunicological perspective Shea discusses four types of reactions and of these type I reactions are "sudden" and may result in "life threatening syptoms" and are generally termed as allergies. ${ }^{3}$ The term food an allergy may be simply defined as "an adverse reaction to food caused by the production of antibodies". ${ }^{4}$ According to Ortolani et al., ${ }^{5}$ food allergy can result in "respiratory, gastrointestinal and cardiovascular symptoms". Allergies tend to have an immediate and severe response and can be life threatening as "symptoms manifest at a rapid rate- anaphylactic shock". ${ }^{5}$

With regard to allergy prevalence, the World Allergy Organisation (WAO) and World Universities Network (WUN) survey was carried out in 2012. This involved the collection of any existing data in an attempt to identify global patterns and prevalence of food allergy and involved "surveying all the member societies of the (WAO) and some neighbouring countries". Of the 89 countries only $10 \%$ had accurate food allergy prevalence data whilst some of the others consisted of parent-reporting as you can see from Figure 1, illustrating the prevalence in pre-school children. Based on more accurate measures, the survey also found "the prevalence of clinical food allergy in preschool children may be as high as $10 \% "{ }^{6}$

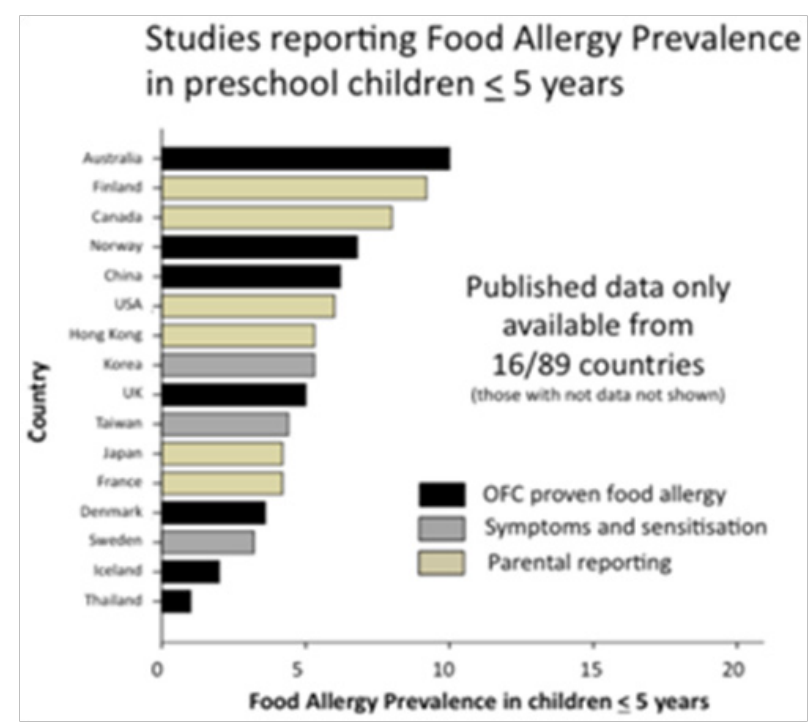

Figure I Summary of food allergy prevalence from studies that provided data for children aged 5years or less. Studies are categorised according to level of evidence; OFC proven food allergy (black bars); food allergy based on symptoms and sensitisation (grey bars) or questionnaires/parental reporting (yellow bars). 
Type IV reactions may be termed as "food sensitivities, chronic delayed food sensitivities" or what we know as food intolerances" and unlike the type I food allergy reactions, they do not include foods which can result in anaphylaxis or death. ${ }^{3}$ Food intolerance is defined as a non-immunological adverse reaction to food, according to Ortolani et al. these reactions are referred to as a "non-allergic food hypersensitivity". ${ }^{5}$ The Irish Nutrition and Dietetic Institute (INDI) state that "an intolerance to food is rarely life threatening and tends to occur hours or days after food is eaten". ' It is important to note that not all food intolerances are non-immunological, for instance celiac disease is an autoimmune disease associated with consumption of the protein gluten and is still classified as an intolerance. Although the symptoms may be milder for food intolerances, they can also be dangerous as symptoms can be silent and can result in inflammation of the digestive tracts; a classic example of this is irritable bowel syndrome (IBS). Food intolerances may also have more atopic symptoms such as hayfever, asthma or eczema, and a study carried out by Young et al found these symptoms were reported in $27 \%$ of its overall respondents. ${ }^{8}$ In addition to this, food intolerances can also result in symptoms such as "nausea, vomiting, prolonged fatigue, sudden or unexplained weight loss and failure to thrive in children" in addition to typical sypmtoms such as irritable bowel and abdominal bloating as well as other symptoms. ${ }^{9}$

Due to the lack of understanding of these mechanisms and ambiguity in the diagnosis of food intolerances in particular, the medical profession often prescribe medications such as anitihistamines, steroid creams and nasals sprays to counteract and mask the symptoms, however the consequences of long term use of such products and interventions must be questioned.

We can establish from the above that there is a great need to gain a further understanding of the various mechanisms involved in allergies and intolerances, this in turn would assist the public, health care professionals and the food industry to appropriately manage the diagnosis, treatment and ensure that the appropriate control systems are put in place.

\section{Causes of food allergies \& intolerances}

With regard to food intolerances, Ortolani et al., ${ }^{5}$ suggests that they may be as a result of a couple of factors including enzyme defect or pharmacological substances present in food and "reactions nonclassifiable in any known mechanism are referred to as undefined food intolerance". ${ }^{5}$ There may be however other potential factors.

Genetic predisposition or familial exposure may give rise to celiac disease which is "characterised by a strong genetic association with the MHC-II haplotype". ${ }^{10}$

Globalisation involving movement of populations and cultures may also have a role to play in the increase of food allergies and intolerances as Haley states "it is not only populations that migrate but also foods, as people adopt foreign diets and import exotic products". We may therefore be exposed to foods which aren't accustomed to.

The processing of foods may have a role to play. According to Ortolani et al., "toxins can be naturally present in foods but induced by processing or incorporated into food as contaminants or additives". They also state that "exogenous toxins may result from food processing and storage or traces of substances used for food production may remain in food as methionine sulphoximine and trichloroethylene". 5 These substances used as bleaching agents and solvent extraction can have serious health implications.
We must question the use of genetic engineering in food production. Although we have an aging population and as a result must explore new techniques to ensure food sustainability and security, the safety, compatibility and use of genetically modified (GMO) foods must be questioned. Within the Euro Prevall report they state that "the lack of effective tools for allergic risk assessment of GMO's intended for food use has already been highlighted by the European Food Safety Authority (EFSA)" and also state that there is a need to "take into account the effects of the food matrix and food processing procedures on allergic potential, which is still poorly understood". Although GMO foods have yet to be proven or disproven, we must still consider their potential role in the increase of intolerances and allergies.

\section{Diagnosis of allergies and intolerances}

One of the main issues that exist regarding food allergies and intolerances is an efficient, reliable and scientific technique for diagnosis. The Euro Prevall report states a "correct diagnosis is essential for the food-allergic patient. Food allergies can result in uncomfortable, severe or potentially fatal responses". ${ }^{11}$

Many individuals choose methods such as cytotoxicity, bioresonance or kinesiology testing however there is little or no scientific evidence to support these techniques.

Provocation/neutralisation testing has been used in practice. This technique uses sublingual drops containing standardised concentrations of the food substrate. According to Shea these drops are "administered one after another until the allergen is identified by symptom provocation". One must consider the safety of this technique as well as it being considered as "expensive and time consuming".

Another technique is that of electrodermal testing, which according to Kennedy et al is a non-invasive bioenergetic evaluation of the body's response to different stressors via measurement of the skin's electrical activity at designated acupuncture points" and measuring the resistance of each of the food items which is digitally recorded via a laptop. Although the study carred out by Kennedy et al found this test to have high sensitivity, its' specificity was only found to be $50 \%$ and therefore not near accurate enough for use within common practice. $^{12}$

According to Ortolani et al. "Skin prick tests (SPT) using commercial extracts are variable but using food in its natural form (prick+prick) is more reliable. In general SPTs remain the tests of first choice". They discuss further that there is a possibility that both can result in false positives, therefore the accuracy of this must be questioned.

The elimination diet is a common and traditional technique suggested to individuals by eliminating one food at a time and keeping a diary. The downside to this is it requires a lot of discipline, effort and can require a lot of time to establish the exact foods which are causing the issue. Ortolani et al. suggest that " 3 weeks are sufficient to confirm the suspect of a food allergy" however this is questionable. ${ }^{5}$

IgE (allergen marker) screening tests are quite efficient and can be read against a broad range of major food allergens and "allows identification of the source of sensitization" suggesting the microarray chip is the way for ward. ${ }^{11}$

Although these are some of the most commonly used and discussed diagnositic techniques there is no doubt that there are more tests available. This in itself heightens the need for gold standard and scientifically reliable diagnostic techniques to be used in common practice. 


\section{Management of food intolerances and allergies}

Unfortunately the only effective management for the patient is the avoidance of the toxin or allergen which is the cause of the issue. As Alan Reilly FSAI chief executive states "Strict attention to the food and ingredients in the foods they choose is a necessity and way of life". ${ }^{2}$

The food industry has a large role to play in ensuring 'free from' food availability and options but also to provide a safe environment for those who have special needs relating to food intolerances and allergies.

\section{Preventative and curative strategies for food allergies and intolerances}

The Euro Prevall report suggests that there is requirements to further understand the mechanisms involved in "sensitisation/ tolerance induction", however it does suggest the potential use of dietary interventions such as "pre and probiotics" and the manipulation of the timing of unsaturated fats. It also mentions that "pre-natal rather than post-natal interventions may be more effective in determining outcomes in infants-epigenetic effects", however the later has yet to be proven. ${ }^{11}$

Also Harrer states that existing knowledge of IgE and T-cell immune reactivity "allows for development of computational algorithms to predict allergenicity of novel foods being introduced by biotechnological industry". ${ }^{13}$

\section{Challenges related to food allergies and intolerances}

Unfortunately the perception of food intolerances and allergies is distorted not only by the public but also by many medical professionals who dismiss many of the diagnostic techniques that are currently used. As Hadley quotes "there are still too many situations where people do not recognise that food allergy is a medical condition, not a food preference". ${ }^{1}$ This has serious implications not only from a diagnostic and social point of view but also within the food industry as the importance of allergen control systems may be underestimated.

\section{The burden of food allergies \& intolerances}

\section{To the individual}

Within the Euro Prevall study, partners based in the Netherlands and Ireland developed "disease specific quality of life measurement instruments" in the form of a suite of questionnaires specific to four groups including children, teenagers, adults and parents of allergic children. Interestingly these revealed that the food avoidance was the primary issue as opposed to the actual severity of reaction. This therefore shows that restriction on the types and variations of food available has a direct effect on quality of life. What may further restrict food options is the cost of 'free from' foods. ${ }^{11}$

For adults and parents of allergic children the cost of treating undiagnosed food intolerances through medication and repeat doctor's visits can be staggering.

In Individuals who have severe diagnosed allergies there is always the fear of cross-contamination with potential unintentional exposure to allergens for instance at children's birthday parties or restaurants.
Both adults and children who have severe food intolerances may get symptoms of inflammation of the digestive tracts and as a result can result in problems with nutrient absorption, and malnutrition. ${ }^{14}$ Such examples of this may be non-sickle cell anaemia, celiac disease and non- celiac gluten sensitivity. In fact the later that is non-celiac gluten sensitivity (NGCS) is a relatively new condition which according to Mansueto, affects a significant percentage of the population who have issues with gluten ingestion without testing positive for celiac disease. $\mathrm{He}$ and his colleagues suggest that "it is very probable that the new kinds of wheat, particularly enriched in gluten content, have greatly contributed to the explosion of gluten-related diseases". ${ }^{15}$ Although further research is required in this field we must still consider this as a possibility.

Labelling of allergens or allergen free foods is also an issue. Within the EuroPrevall report they state that "allergic consumers experience stress as a result of poor communication and labelling practices". For instance it was found that supermarkets with higher prices were more likely to adhere to E.U. standards where as supermarkets selling products at a lower price did not meet E.U. standards. In addition to this consumers found that the information was "overwhelming and presented in too small a font size" and that "these needs conflict with the desire for food allergic consumers to have complete information about the products they buy". ${ }^{11}$

The social aspect of food allergies and intolerances can also result in social implications in that individuals may fear eating out or fear of social exclusion. In fact an interesting study by Bollinger et al looks at the impact of food allergy on the daily activities of children and their families. Within this study it was found that " $49 \%$ or more indicated that food allergy affected family social activities, 34\% reported that allergy had an impact on school attendance and 10\% choosing to home school their children because of food allergy". ${ }^{16}$

\section{To food manufacturers}

Although food allergen control may affect workload, legislative procedures, quality control and production costs this should be seen as an opportunity. As the requirement for allergen free foods is on the rise, the food industry is taking advantage of this new market allowing for new product development and innovation and the provision of these 'specialised' foods at a higher cost. In fact in a Bord Bia state that "in 2013 , sales of gluten-free products totalled US $\$ 181.2 \mathrm{~m}$, representing a compound annual growth rate of $12 \%$ from 2008 with gluten-free baby food accounting for $84 \%$ of the total gluten-free market". ${ }^{17}$ From a food business perspective this is certainly a profitable area.

\section{Responsibilities of the food industry}

It is necessary for the food industry to adapt to the needs of the consumers with regard the production of a higher variety of 'free from' foods but also in relation to quality control systems which include hygiene, labelling and contaminant control.

Alan Reilly FSAI chief executive stated in his most recent report that "even amongst experienced food business operators, who are required to put allergen labelling on foods, mistakes occur", he also stated that "recalls and withdrawals of packaged foods for this reason average two per month". It is for this reason that strict quality control protocols need to be followed to prevent "failure in the review of product labels, use of incorrect packaging, inadequate separation of foods or tools during manufacture or failure to check supplier certificates". ${ }^{2}$ 
For food manufacturers who choose to produce allergen free food it is imperative for them to become familiar with the manufacture requirements and the development of allergen control systems and protocols such as storage of raw ingredients, food handling and production. They must also take due care to ensure that labelling is accurate and to precautionary labelling used where necessary. In fact in Ireland from December 2014 the labelling rules that currently exist for pre-packaged food will be extended to 'loose food' which according to the FSAI are "unpackaged foods or drinks sold directly to consumers from shops, stalls, canteens, restaurants and virtually all food premises". What this essentially means is that "clear information about the fourteen prescribed allergenic foods will have to be available in written format at the point of presentation, sale or supply". ${ }^{2}$ These new requirements may help to increase consumer confidence with regard to the safety of their food.

Obligatory training programmes throughout the food industry and for food handlers is necessary to illustrate the importance of maintaining these standards and the hazards associated with allergen contamination may prove useful in heightening the awareness of consequences of not following protocol.

\section{Responsibilities of the individual}

It is imperative for those who are diagnosed and aware of their allergies and intolerances to actively control their risk of exposure. Firstly it is important to receive correct and accurate information and education from the healthcare providers who provide the diagnosis. This should include information regarding food allergens and contaminant control within the kitchen and when eating out. It is then the responsibility of the individual to research food products and restaurants to ensure that they have 'free from' options or recipes to suit their particular needs. Once the information has been provided a certain amount of responsibility then lies with the individual to enforce these changes within their own lives so as to prevent their own potential exposure to risk where possible.

\section{Recommendations}

The following recommendations have been suggested using existing and current evidence and research including the EuroPrevall cohort study and FSAI guidelines. ${ }^{2,11}$

Gold standard scientific diagnosis techniques for the both food intolerance and food allergies need to be established which are cost effective and accessible to the wider public. Provision of information to the public about recognising the underlying symptoms to ensure that those with undiagnosed intolerances and allergies are get diagnosed before the symptoms cause serious health implications.

Public education and training is required explaining food intolerances and allergies, the risks associated and how comfortably adapt food habits and techniques to meet these requirements to without having a negative effect on their health or quality of life.

Food production and quality control measures need to be effectively enforced for food producers and food outlets. This may involve changes of allergen control systems and training of staff to remove any uncertainty with regard to protocol or procedures when dealing with allergens.

We are still unsure about allergen threshold concentrations, at which there is an "acceptable level of protection for at risk customers". ${ }^{2}$ The FSAI have posed this question to the European Food Safety Authority (EFSA) for clarification.
It will be imperative to ensure the enforcement of the new requirement for loose and unpackaged foods as well as restauant menus to contain "clear information regarding the 14 prescribed allergenic foods at the point of presentation, sale or supply, extending to distance selling via websites or catalogues".

To conclude further research needs to be done into the mechanisms, diagnosis, prevention and protection against food allergies and intolerances to prevent a further increase and protect the public from unnecessary harm.

\section{Acknowledgements}

None.

\section{Conflict of interest}

Author declares that there is no conflict of interest.

\section{References}

1. Hadley. Food allergies on the rise? Determining the prevalence of food allergies and how quickly it is increasing is the first step in tackling the problem. EMBO Rep. 2006;7(11):1080-1083.

2. FSAI. Food Allergens- More to be Revealed. Fsai News. 2014;16(5):1.

3. http://www.google.com/patents/US8802056

4. Bender D. Oxford Dictionary of Food \& Nutrition. USA: Oxford University Press; 2005.

5. Ortolani C, Pastorello EA. Food allergies and food intolerances. Best Pract Res Clin Gastroenterol. 2006;20(3):467-483.

6. Prescott SL, Pawankar R, Allen KJ, et al. A global survey of changing patterns of food allergy burden in children. World Allergy Organ J. 2013;6(1):21.

7. INDI. Food Allergies and Intolerances-Diseases Allergies and Medical Conditions. Irish Nutrition \& Dietetics Institute. 2013.

8. Young E, Stoneham MD, Petruckevitch A, et al. A population study of food intolerance. Lancet. 1994;343(8906):1127-1130.

9. NICE. Coeliac Disease: recognition and assessment of coeliac disease. 2009.

10. Mansueto P, Seidita A, D'Alcamo A, et al. Non-Celiac Gluten Sensitivity: Literature Review. J Am Coll Nutr. 2014;33(1):39-54.

11. EuroPrevall. The Prevalence, cost and basis of food allergy across Europe-Final report summary. BBSRC Institute of Food Research. 2010 .

12. Kennedy D, Lewis E, Cooley K. An exploratory comparative investigation of Food Allergy/Sensitivity Testing in IBS (The FAST Study): A comparison between various laboratory methods and an elimination diet. Advances in Integrative Medicine. 2014;1:124-130.

13. Harrer A, Egger M, Gadermaier G, et al. Characterization of plant food allergens: An overview on physicochemical and immunological techniques. Mol Nutr Food Res. 2010;54(1):93-112.

14. Meyer R, De Koker C, Dziubak R, et al. Malnutrition in children with food allergies in the UK. J Hum Nutr Diet. 2014;27(3):227-235.

15. Mansueto P, Seidita A, D'Alcamo A, et al. Non-Celiac Gluten Sensitivity: Literature Review. J Am Coll Nutr. 2014;33(1):39-54.

16. Bollinger ME, Dahlquist LM, Mudd K, et al. The impact of food allergy on the daily activities of children and their families. Ann Allergy Asthma Immunol. 2006;96(3):415-421.

17. BordBia. Food alert capturing the trend in the global food and drinks market. Bord Bia-Irish Food Board; 2014. 